\title{
Numerical study of heat transfer in rectangular channels with single pin fin and pin fin-dimple
}

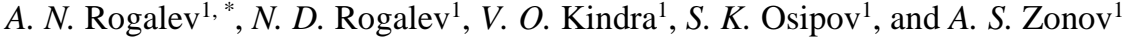 \\ ${ }^{1}$ National Research University "Moscow Power Engineering Institute", Moscow, Russia
}

\begin{abstract}
Evaluation of the heat transfer and hydraulic performance of a new pin fin-dimple cooling system in a rectangular channel shows its advantage. The performance are compared with the pin fin system ones with 3-D Reynolds averaged Navier-Stokes (RANS) equations. The fluid flow and heat transfer analysis for the Reynolds numbers from 8000 to 70000 involved the shear stress transport turbulence model. The new system forms a high-intensity vortex around the pin fin-dimple that increases the near-wall turbulent mixing level that intensifies the heat transfer. The calculation results indicate increases of the averaged Nusselt number and the averaged friction factor of $7-13 \%$ and $7-12 \%$ respectively against the pin fin.
\end{abstract}

\section{Introduction}

One of the main methods for convection heat transfer intensification is the application of flow turbulators. Pin fins, ribs, dimples or swirl matrixes located in channels and on coolant blown surfaces are widely used [1-4]. These elements destruct wall flow layers, introduce flow compound motions and thus intensify the heat transfer.

The pin fins remarkably increase the surface heat release. The largest intensification is obtained in the turbulator frontal part, and there may be seen a stagnation zone with low heat transfer coefficients in the turbulator trailing part. Experimental relations between the Nusselt and Reynolds numbers in straight and narrowing channels with corridor and staggered pin fin array locations and different ratios of geometry parameters are given in [5-8]. Influence of a single pin fin shape upon the flow swirl structure and the channel thermal performance is disclosed in [9].

The pin fin-dimple staggered array [10] is a modification of the staggered pin fin array that allows a considerable increase of the surface heat release.

The experimental data comparison in the Reynolds number range of 4000-14000 showed that, compared with pin fin channel, the pin fin-dimple channel has increased convective heat transfer performance and flow friction by $11 \%$ and $9 \%$ respectively [11].

This study presents comparative computation results for two single heat transfer intensifiers located in a rectangular section cooling channel. The thermal and hydraulic analysis results are shown for a channel with the flow turbulator, the heat transfer intensification is explained on the base of swirl flow structure.

\section{Description of the research object}

The investigation subject is a rectangular section cooling channel with a single pin fin or pin fin-dimple heat transfer intensifier. For calculation of the considered turbulator specific thermal performance, a smooth channel was also investigated. Dimensions of the channel and the single flow intensifiers are shown in figure 1 .

These values of the channel length $L$ and the distance from channel inlet to intensifier center $N$ allow simulations free from the channel inlet and exit conditions. The selected channel width $B$ eliminates the side walls boundary layers influence upon the thermalhydraulic process in the cooling air flow around the intensifiers. The channel height $H$ provides the ratio $H / D_{\text {pin }}=1$. Hydraulic diameter is equal to $3.4 \mathrm{~mm}$.

\section{Method for the thermal-hydraulic process computer simulation}

In the present study, 3-D RANS analysis of the fluid flow and convective heat transfer were performed using the ANSYS-CFX software. The non-structured mesh was built for the main flow with the ANSYS ICEM code. The main flow mesh elements are tetrahedrons; the wall region mesh is formed by prismatic layers. The mesh parameters are equal for all models and are summarized in table 1.

The Reynolds averaging Navier-Stocks (RANS) equations are closed with the SST turbulence model. The working fluid was air. Conditions of the computer simulation test are summarized in table 2 .

\footnotetext{
Corresponding author: r-andrey2007@yandex.ru
} 


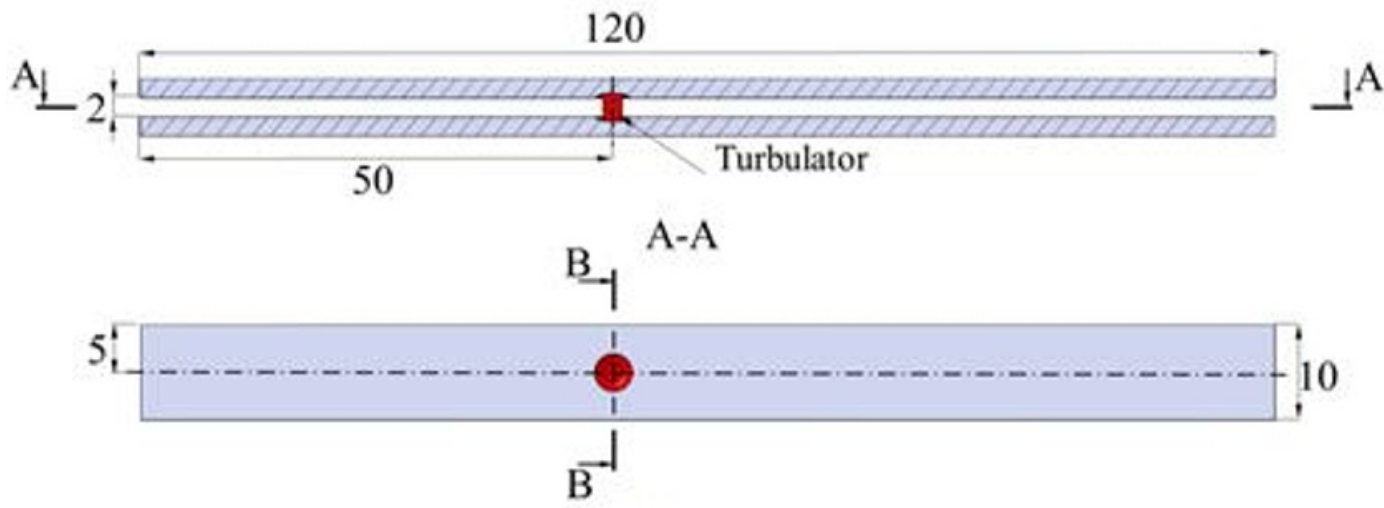

a)

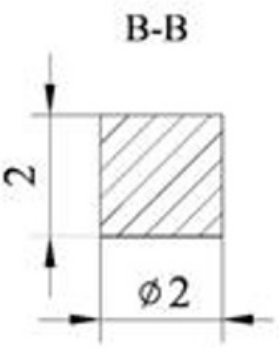

b)

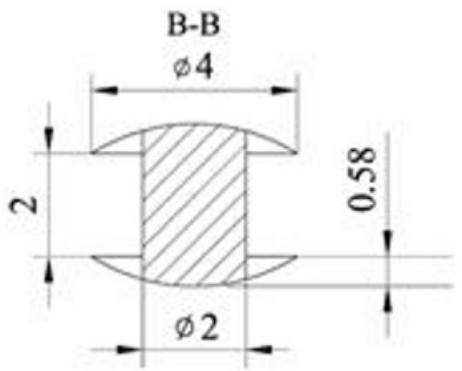

c)

Fig. 1. Dimensions of the channel with single heat transfer intensifiers.

Table 1. Mesh parameters.

\begin{tabular}{|c|c|}
\hline Parameter & Value \\
\hline Global element, $\mathrm{mm}$ & 0.45 \\
\hline Initial height, $\mathrm{mm}$ & 0.009 \\
\hline Height ratio & 1.2 \\
\hline Numbers of layers & 11 \\
\hline Total height, $\mathrm{mm}$ & 0.36 \\
\hline Total elements, $\mathrm{M}$ & 4.9 \\
\hline
\end{tabular}

Table 2. Conditions for the computer simulation test.

\begin{tabular}{|c|c|c|c|c|}
\hline Parameter & \multicolumn{4}{|c|}{ Value } \\
\hline Reynolds number, Re & 8000 & 14000 & 30000 & 70000 \\
\hline $\begin{array}{c}\text { Cooling air massflow, } \\
G, \mathrm{~g} / \mathrm{s}\end{array}$ & 0.88 & 1.6 & 3.3 & 7.7 \\
\hline $\begin{array}{c}\text { Cooling air total } \\
\text { temperature at the } \\
\text { channel inlet, } T_{\text {in }},{ }^{\circ} \mathrm{C}\end{array}$ & \multicolumn{3}{|c|}{20} \\
\hline $\begin{array}{c}\text { Channel internal } \\
\text { surface temperature } \\
T_{\text {wall, }}{ }^{\circ} \mathrm{C}\end{array}$ & \multicolumn{4}{|c|}{419} \\
\hline $\begin{array}{c}\text { Temperature factor } \\
T_{\text {wall }} / T_{\text {in }}\end{array}$ & \multicolumn{4}{|c|}{2.4} \\
\hline $\begin{array}{c}\text { Cooling air static } \\
\text { pressure at the } \\
\text { channel exit } p_{\text {out }} \text {, bar }\end{array}$ & \multicolumn{4}{|c}{1} \\
\hline
\end{tabular}

In all considered versions the cooling airflow static pressure at the channel exit, the channel wall temperature and the cooling air total pressure at the channel inlet were taken constant. Transitions to new flow performance were done by changes of the input massflow at the channel inlet. The parameters values for computer simulation of the single turbulator were near the physical liquid metal bath test [12].

\section{Single heat transfer intensifier, results analysis}

Figure 2 and figure 3 show distributions of the Nusselt number along the channel length, smooth and with different single intensifiers. Each channel may be split into a few zones of flow regime.

The smooth channel shows two zones, the zone of flow inlet conditions influence $(0<L / d<10)$ and the stable flow one $(10<L / d<36)$. The channels with intensifiers show three zones, the zone of flow inlet conditions influence $(0<L / d<10)$, the stable flow zone $(10<L / d<14$, $22<L / d<36)$ and the intensive heat transfer zone $(14<L / d<22)$. These zones borders may be slightly different for each heat transfer intensifier at different Reynolds number values.

The single pin fin application increases the Nusselt number in the mentioned zone for $50-80 \%$ at Reynolds numbers range from 8000 to 70000 due to the flow turbulization. The distribution of heat flow density $q$ (figure 4a) qualitatively confirms the heat release increase in the frontal, or ram part of the intensifier, and in its "shadow" zone there is a low heat transfer stagnation area. 


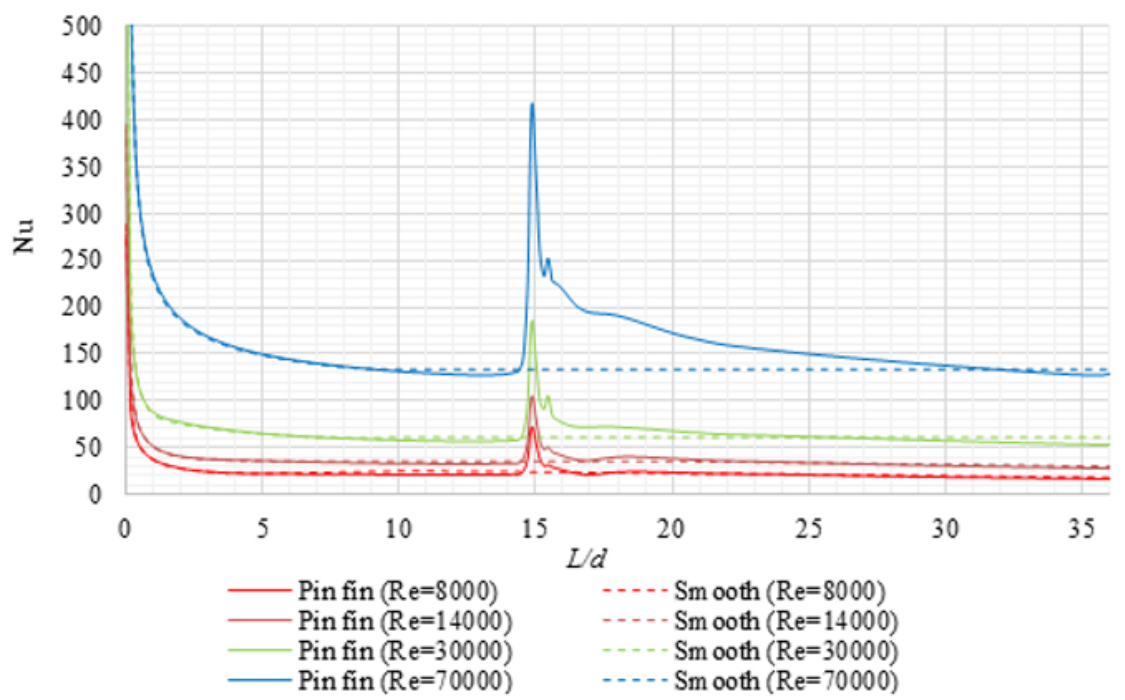

Fig. 2. Influence of Reynolds number upon the Nusselt number distribution along the smooth channel and the channel with a single $D_{\text {dimp }}=2 \mathrm{~mm}$ diameter pin.

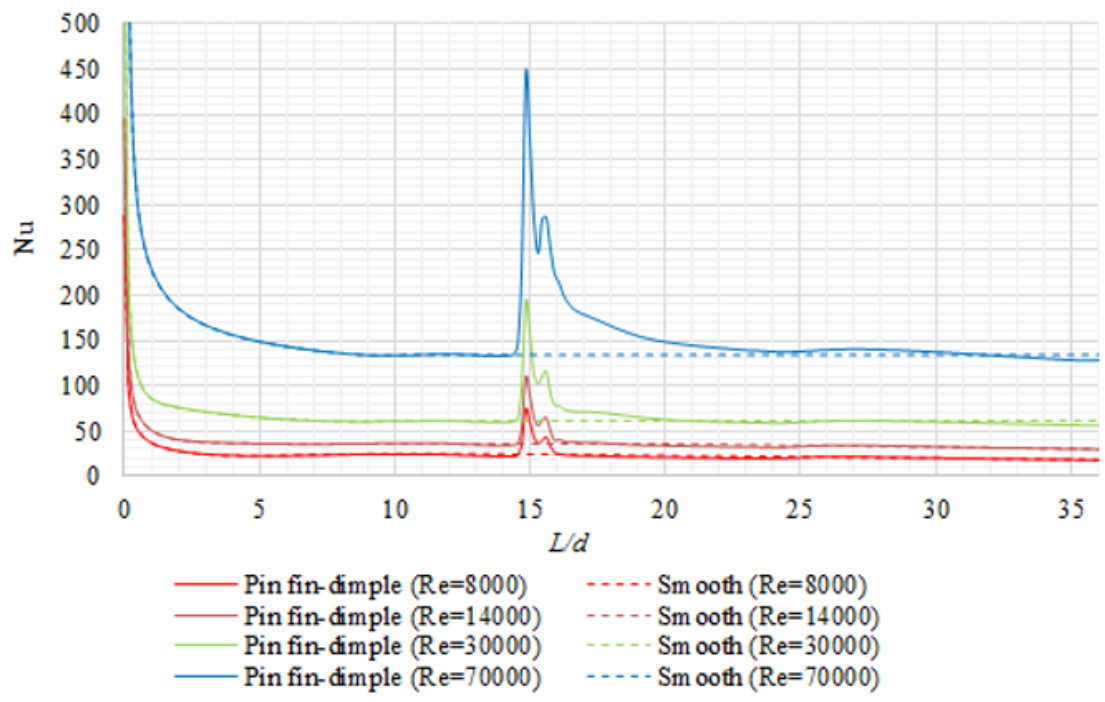

Fig. 3. Influence of Reynolds number upon the Nusselt number distribution along the length of the smooth channel and channel with a single pin fin-dimple intensifier (pin diameter $D_{\text {pin }}=2 \mathrm{~mm}$, dimple diameter $D_{\text {dimp }}=4 \mathrm{~mm}$, pin fin-dimple intensifier depth $\left.h_{\text {dimp }}=0.58 \mathrm{~mm}\right)$.

The single pin fin-dimple application increases the Nusselt number for $60-100 \%$ at the whole range of Reynolds numbers. The heat release distribution around this turbulator at Reynolds number 14000 is shown in figure $4 \mathrm{~b}$. Almost the whole zone in the dimple around pin fin shows the high heat flow intensity.

The channel flow streamlines in figure 5 shed light on the heat transfer intensification by application of the

Table 3. Averaged thermal-hydraulic performance of channels with different heat transfer intensifiers

\begin{tabular}{|c|c|c|c|c|c|}
\hline $\operatorname{Re}$ & $\mathrm{Nu}$ & $\mathrm{Nu} / \mathrm{Nu}_{0}$ & $f$ & $f / f_{0}$ & $\eta$ \\
\hline \multicolumn{6}{|c|}{ Channel with a pin fin } \\
\hline 8000 & 36 & 1.5 & 0.47 & 5.8 & 0.83 \\
\hline 14000 & 55 & 1.5 & 0.44 & 6.5 & 0.82 \\
\hline 30000 & 99 & 1.6 & 0.42 & 7.3 & 0.83 \\
\hline 70000 & 243 & 1.8 & 0.43 & 8.2 & 0.90 \\
\hline \multicolumn{6}{|c|}{ Channel with a pin fin-dimple intensifier } \\
\hline 8000 & 40 & 1.6 & 0.47 & 5.8 & 0.91 \\
\hline 14000 & 60 & 1.7 & 0.48 & 7.0 & 0.88 \\
\hline 30000 & 108 & 1.8 & 0.47 & 8.0 & 0.88 \\
\hline 70000 & 264 & 2.0 & 0.46 & 8.8 & 0.95 \\
\hline
\end{tabular}




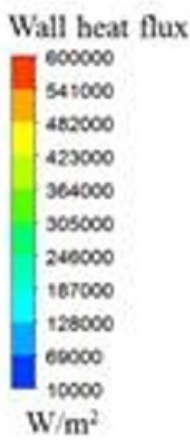

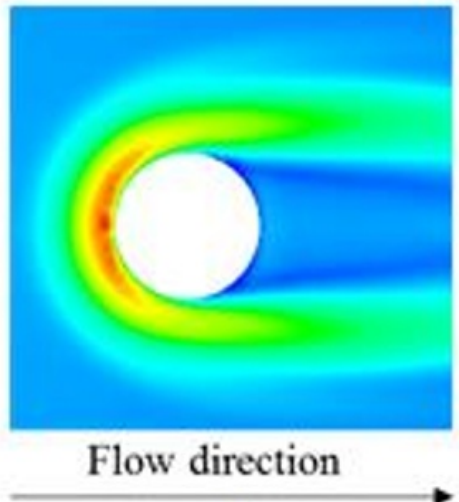

a)

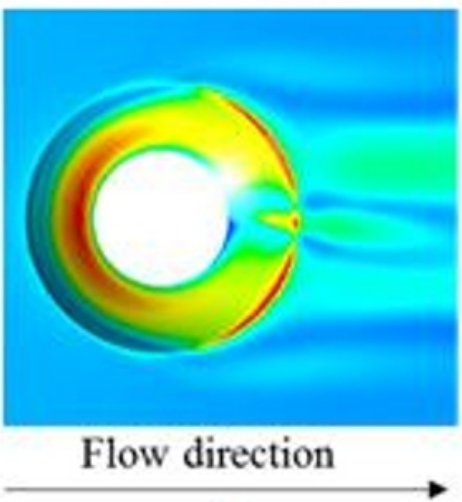

b)

Fig. 4. Heat flow intensity distribution in a channel with single heat transfer intensifiers at $\operatorname{Re}=14000$ : a) Pin fin $\left(D_{\text {pin }}=2 \mathrm{~mm}\right)$; b) Pin fin-dimple $\left(D_{\text {pin }}=2 \mathrm{~mm}, D_{\text {dimp }}=4 \mathrm{~mm}, h_{\text {dimp }}=0.58 \mathrm{~mm}\right)$.

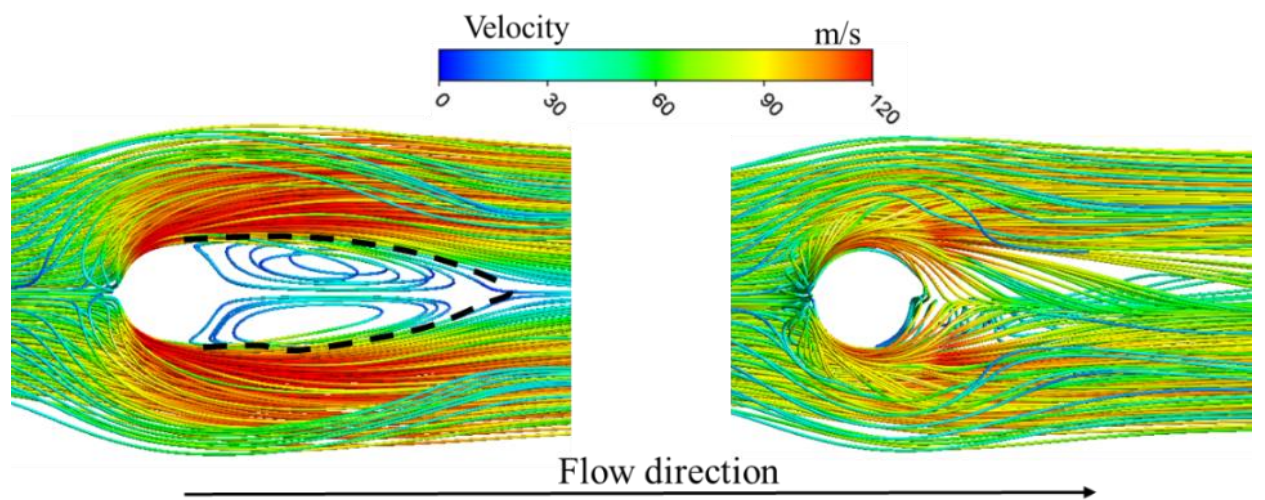

Fig. 5. Flow streamlines for the single pin and pin fin-dimple heat transfer intensifiers.

pin fin-dimple. In the case of pin fin-dimple, the secondary flow structure is complicated. Near the pin fin frontal part, the flow is partly directed into the dimple. The flow axial velocity drops down to zero and then changes its sign. In the dimple ram part there appears a high intensity swirl flow. The pressure drop between the intensifier's frontal and rear parts is remarkable so the swirl flow gets a remarkable axial velocity and is spread through the dimple surface. Finally, the heat transfer in this zone is maximal and the "shadow" zone that is seen in the pin fin is absent in the pin fin-dimple one.

\section{Conclusion}

Computer simulation of single heat transfer intensifiers shows advantages of the pin fin-dimple against the pin fin one. Specifically, in a rectangular cross-section channel fitted with the pin fin-dimple turbulator, the Nusselt number is $7-13 \%$ higher than in the similar channel with the pin fin one. In the case of pin findimple turbulator, the hydraulic resistance is $7-12 \%$ higher.

The heat transfer intensification by pin fin-dimple turbulator is due to the creation of high intensity swirls in the dimple around the pin fin.

The prospective direction for further investigations is the analysis of the main pin fin-dimple geometrical parameters ratios and their influence upon the thermal- hydraulic channel performance and the swirl flow structure.

This study conducted by Moscow Power Engineering Institute was financially supported by the Ministry of Science and Higher Education of the Russian Federation (project No. 13.7616.2017/8.9).

\section{References}

[1] E.Y. Choi, Y.D. Choi, W.S. Lee, J.T. Chung, J.S. Kwak, Heat transfer augmentation using a ribdimple compound cooling technique Appl. Therm. Eng. 51(1-2), 435-441(2013)

[2] J.C. Han, S. Dutta, S. Ekkad, Gas turbine heat transfer and cooling technology (Boca Raton: CRC Press) 843 (2012)

[3] S.A. Isaev, A.I. Leont'ev, Numerical simulation of vortex enhancement of heat transfer under conditions of turbulent flow past a spherical dimple on the wall of a narrow channel High Temperature 41(5), 665-79 (2003)

[4] I.V. Shevchenko, I.V. Garanin, A.N. Rogalev, V.O. Kindra, V.P. Khudyakova, Study of design and technology factors influencing gas turbine blade cooling J. Phys.: 891(1), 012253 (2017)

[5] D.E. Metzger, W.B. Shepard, S.W. Haley, Row resolved heat transfer variations in pin-fin arrays 
including effects of non-uniform arrays and flow convergence ASME Int. Gas Turbine Conf. and Exhibit (Dusseldorf / ASME) (1986)

[6] M.K. Chyu, Y.C. Hsing, T.I. Shih, V. Natarajan, Heat transfer contributions of pins and endwall in pin-fin arrays: effects of thermal boundary condition modeling Proc. Int. Gas Turbine and Aeroengine Congress and Exhibition (Stockholm / ASME) (1998)

[7] D.E. Metzger, R.A. Berry, J.P. Bronson, Developing heat transfer in rectangular ducts with staggered arrays of short pin fins J. Heat. Transf. 104(4), 700-06 (1982)

[8] G.J. VanFossen, Heat transfer coefficients for staggered arrays of short pin fins J. Eng. Power. 104(2), 268-74 (1982)

[9] M.A. Moon, K.Y. Kim, Analysis and optimization of fan-shaped pin-fin in a rectangular cooling channel Int. J. Heat. Mass. Tran. 72, 148-62 (2014)

[10] V.O. Kindra, A.N. Rogalev, S.K. Osipov, I.V. Shevchenko, Development and experimental study of the high efficient flow turbulators for heat transfer enhancement in heat exchangers J. Phys.: Conf. Ser. 1128(1), 012024 (2018)

[11] V. Kindra, S. Osipov, D. Kharlamova, I. Shevchenko, An experimental and numerical study of flow and heat transfer in cooling channels with pin findimple and pin fin-groove arrays Proc. 13th European Conf. on Turbomachinery Fluid Dynamics \& Thermodinamics (Lausanne / ETC) (2018)

[12] I.V. Shevchenko, A.N. Rogalev, M.I. Shevchenko, A.N. Vegera, Method of calorimetric measurements in molten metal thermostat and its application for developing blade cooling system of gas turbines Int. J. Appl. Res. 12(10), 2382-86 (2017) 